\title{
Das Verhältnis von Theologie umd Naturwissenschaft als systematisch-theologissches Problem
}

\author{
Prof. Dr. Hans Schwarz, 583 S. James Rd..., Columbus, Ohio 43213, USA
}

Die Verhältnisbestimmung von Theeologie und Naturwissenschaft konnte erst dann zu einem Problem werrden, als die Naturwissenschaft begann, sich aus dem bis dahin allumfasssenden Wissenschaftsbereich der Theologie auszugliedern um selbständig : zu werden. Diese Verselbständigung deutet sich etwa bei Thomas von $ı$ Aquino an, wenn er zwischen dem natürlichen und dem übernatürlichıen Bereich scheidet ${ }^{1}$, oder bei Bonaventura, der von einem cursus naturalis und einem cursus supernaturalis sive mirabilis redet ${ }^{2}$. Bei Galileii ist dann diese Unterscheidung bereits zu einem Gegensatz geworden, weenn er 1613 in einem Brief fordert, daß die Theologen die Bibel endlicth in Ubereinstimmung mit den neuen Ergebnissen der Wissenschaft interrpretieren sollen'. Zwar bahnte sich in der Physikotheologie des beginneneden 18. Jahrhunderts nochmals eine Synthese von Theologie und Naturearkenntnis an, doch kam es im 19. Jahrhundert unter Einfluß der idealistrischen und dialektischen Philosophie etwa Kants und Kierkegaards zu ceinem endgültigen Bruch. Mitverursacht durch die dialektische Theologie herrscht seit den zwanziger Jahren dieses Jahrhunderts eine eigentiümliche Neutralität zwischen Theologie und Naturwissenschaft, die erstt heute zu weichen beginnt ${ }^{4}$. In dieser Neutralität drückt sich trotz allem Analogiedenkens eine letzte Zweigleisigkeit des Denkens aus, die einee echte Verhältnisbestimmung von Theologie und Naturwissenschaft erfiordert, falls der Riß zwischen beiden Disziplinen nicht noch breiter werdden soll. In der heutigen Theologie gibt es vier Haupttendenzen, dieses Verhältnis zu bestimmen. $\mathrm{Da}$ ist zunächst von einer dialektischen Trenınung zu reden, dann in Fortführung davon von einer nichttheistischen 1 Anknüpfung, sodann von einer religiösen Anknüpfung und schließlich vom einer dimensionalen Verhältnisbestimmung.

\section{Das Verhältnis von Theologie und Naturwissenschaft in der gegenwärtigen Theeologie}

Die dialektische Trennung: Die dialekttische Trennung von Theologie und Naturwissenschaft wird besonders; durch Karl Barth vollzogen. Gegen das, was die Heilige Schrift und die cchristliche Kirche unter Gottes

1 Siehe Thomas, S Th III, 13, 2c (XI, 173, 3).

2 So Bonaventura II d 18 a 1 q 2 ad 5 (II, 437 f.'); vgl. zu Bonaventura die instruktive Arbeit von J. Ratzinger: "Der Wortgebrausch von Natura und die beginnende Verselbständigung der Metaphysik bei Bonavventura «, in: Die Metaphysik des Mittelalters, Berlin 1963, 483--498, hier S. 485.

' Nach E. Dinkler, Max Planck und die Religion, ZThK, 56. Jg., Tübingen 1959, 219. 
Schöpfungswirken verstehen, kann es nach Barth "schlechterdings keine naturwissenschaftlichen Fragen, Einwände und Hilfestellungen geben « ${ }^{5}$. So geht bezüglich der Lehre von Gott dem Schöpfer "die christliche Dogmatik der eigenen Aufgabe nach... Wenn ihr die Weltanschauungen dabei nicht helfen können, so wird sie sich durch diese auch nicht stören lassen " ${ }^{6}$. Genauso wie die Theologie ihren eigenen, unabhängigen Arbeitsbereich hat, wird er auch der Naturwissenschaft zugestanden, denn "die Naturwissenschaft hat freien Raum jenseits dessen, was die Theologie als Werk des Schöpfers zu beschreiben hat « ${ }^{7}$. Zu einem echten Dialog kann es schon deswegen nicht kommen, weil Barth mit Hilfe der Analogia Fidei schon alles, was man über die Welt aussagen kann als Theologie aus Gott und aus seiner Offenbarung deduziert. Barth hat berechtigterweise die Theologie wieder auf ihre eigentliche Hauptaufgabe zurückverwiesen, auf das reflektierende Nachsagen dessen, was uns durch die Offenbarung vor- und zugesagt wurde. Doch vernachlässigt er dadurch, daß er ständig auf Gott als dem Subjekt, Prädikat und Objekt der Offenbarung pocht, daß sich die Offenbarung in Raum und Zeit ereignet hat. Er hält zwar gegenüber jedem Spiritualisierungsversuch fest, daß die Historizität Jesu heilsnotwendig ist ${ }^{8}$. Aber wenn er andererseits den historischen Jesus für belanglos erklärt ${ }^{\circ}$, so scheint das Wo dieser Offenbarung, also die Welt, nicht ebenso ernst genommen zu werden wie das Wer der Offenbarung, also Gott. Damit verflüchtigen sich die Phänomene der Welt und Geschichte $^{10}$; die Welt wird allein gelassen ${ }^{11}$, denn sie kommt gegen die Theologie nicht $\mathrm{zu}$ Wort, und geht daher ihre eigenen und vielleicht der Theologie entgegengesetzten Wege.

Die nichttheistische Anknüpfung: Wird bei der dialektischen Trennung von Theologie und Naturwissenschaft die Unterschiedenheit beider

4 Vgl. dazu auch G. Altner, Schöpfungsglaube und Entwidklungsgedanke in der protestantischen Theologie zwischen Ernst Haeckel und Teilhard de Chardin, Zürich 1965, 83.

5 Siche K. Barth, KD III/1, Vorwort.

- Siehe Barth, KD III/1, 394.

7 Siehe Barth, KD III/1, Vorwort.

8 Vgl. Barth, KD III/2, 529.

- Vgl. K. Barth, Das Wort Gottes und die Theologie, München 1925, 95, wo er sagt: »Mag es sich mit dem historischen Jesus verhalten, wie es will, Jesus der Christus, des lebendigen Gottes Sohn, gehört weder der Historie noch der Psychologie an; denn was historisch und psychisch ist, das ist eben als solches auch verweslich *.

10 So wendet besonders G. Rödding, Das Seinsproblem in der Schöpfungslehre Karl Barths, KuD, 10. Jg., Göttingen 1964, 41, gegen Barth ein, daß sich die Phänomene von Welt und Geschichte deswegen verflüchtigen, da der Gottesbegriff zum Grundbegriff der Dogmatik schlechthin wird und da sich die Welt eben nicht aus Gott deduzieren läßt.

11 Wenn D. Bonhoeffer, Widerstand und Ergebung, Briefe und Aufzeichnungen aus der Haft, hg. v. E. Bethge, München 1958, 185, Barth berechtigterweise einen Offenbarungspositivismus vorwirf, so wendet er dabei besonders gegen das Vorgehen Barths ein, daß die Welt sich selbst überlassen wird. 
Gebiete so betont, daß eine positive Verhältnisbestimmung unmöglich wird, und die Naturwissenschaft letztlich nicht ernst genommen wird, so erfolgt mit der nichttheistischen Anknüpfung das Gegenteil. Die Welt und die Ergebnisse der Naturwissenschaft werden in einer bisher noch nicht dagewesenen Weise als allgemeinverbindliche und allgemeingültige Wirklichkeit akzeptiert. So wendet sich etwa Bonhoeffer strikt dagegen, daß Gott zu einem Lückenbüßer degradiert wird ${ }^{12}$. Gott ist nicht erst an den Grenzen unseres Lebens zu finden, sondern er muß mitten im Leben erkannt werden. Dabei ist zwar nicht alles, was geschieht, mit Gottes Willen gleichzusetzen, aber es geschieht nichts ohne seinen Willen ${ }^{13}$, denn durch jedes Ereignis hindurch findet man den Weg zu Gott. Bonhoeffer hat die Religion, die bei Barth noch die sündhafte Grundbefindlichkeit des Menschen ist, als theistische "Antenne" des Menschen gestrichen, wenn er erklärt, daß sie nur ein geschichtliches, vielleicht nicht mehr wiederkehrendes westliches Phänomen ist ${ }^{14}, \mathrm{da}$ Gott uns in der Welt so leben läßt, etsi deus non daretur ${ }^{15}$. Aber indem Gott auch mitten im Leben jenseitig $\mathrm{da}$ ist, wird bei der sogenannten nichtreligiösen Interpretation Bonhoeffers, bei der die Religion keine Möglichkeit des Menschen mehr ist, die Gegenwart Gottes in der Welt vorausgesetzt ${ }^{16}$. Bonhoeffer hielt dabei zwar am Christozentrismus Barths fest ${ }^{17}$, doch setzte er mit dieser Interpretationsweise die Theologie mit der Welt in Beziehung ${ }^{18}$ und ermöglichte so ein Gespräch mit der Naturwissenschaft unter Wahrung der Eigenständigkeit beider Partner. Indem er aber das religiöse Apriori als geschichtliches Phänomen beseitigte, begab er sich in gewisser Weise auf die gleiche, von der Naturwissenschaft abhängige Argumentationsbasis wie Bultmann, als dieser erklärte, daß man angesichts unseres technischen Fortschritts die Wunderwelt des Neuen Testaments nicht mehr akzeptieren könne ${ }^{19}$. Dies führt dann etwa bei Herbert Braun dazu, daß er im Gegensatz zu Bultmann und Bonhoeffer auch das personale Gegenüber Gottes als unnötige Voraussetzung der christlichen Theologie aufgab ${ }^{20}$

12 Zum Folgenden vgl. Bonhoeffer, Widerstand, 211. Wiewcit man natürlich diese Briefe aus dem Gefängnis legitim systematisch-theologisch auswerten darf, ist eine andere Frage. Aber wegen des großen Einflusses, den sie ausübten und immer noch ausüben, müssen wir auf sie eingehen.

13 Ahnlich Bonhoeffer, Widerstand, 120.

1" Vgl. Bonhoeffer, Widerstand, 178 f., und E. Bethge, Dietrich Bonhoeffer, München 1967, 978 f., der hier auf den Unterschied zu Barth ausführlich hinweist.

${ }^{15} \mathrm{H}$. Ott, Wirklichkeit und Glaube, Erster Band, Zum theologischen Erbe Dietrich Bonhoeffers, Zürich 1966, 37, meint hier, daß Bonhoeffer es gelang, den Personalismus Gollwitzers und die Mitmenschlichkeit Brauns gleichzeitig zu wahren.

16 So auch Ott, Wirklichkeit, 124.

17 Dies betont Ott, Wirklichkeit, 113.

18 Ahnlich auch R. Prenter, Dietrich Bonhoeffer und Karl Barths Offenbarungspositivismus, in: Die mündige Welt, III Bd., München 1960, 17.

$29 \mathrm{Vgl}$. R. Bultmann, KuM, Bd. 1, Hamburg 19604, $17 \mathrm{f}$.

so Vgl. H. Braun, Gottes Existenz und meine Geschichtlichkeit im Neuen Testament, 
und die Offenbarung gemäß dem geistigen Aspekt des jeweiligen Menschen interpretiert ${ }^{21}$. Auch wenn hier das Motiv dahintersteht, daß die Souveränität Gottes keinerlei Vorbedingung braucht ${ }^{22}$, kein religiöses Apriori und keine theistische Personstruktur, so ist man doch hier in solche Abhängigkeit von der Naturwissenschaft gekommen, daß man die von der Naturwissenschaft aufzeigbare Wirklichkeit für die alleingültige hält. Damit ist kein echtes Gespräch mehr möglich, denn Vorgabe und Ergebnis des Gesprächs kann jeweils nur das sein, was im Verstehenshorizont der Naturwissenschaft liegt. Auch die Strömungen der angelsächsischen Theologie, die zum Teil hier geistesgeschichtlich parallellaufend, Gott in den natürlichen Ursachen, im sozialen Wandel oder in seiner Abwesenheit und im Leiden empfinden ${ }^{23}$, zeigen von ähnlicher Abhängigkeit von naturwissenschaftlichen Weltanschauungen und gleicher Unfähigkeit, zu einer echten Verhältnisbestimmung $z$ wischen den Erkenntnissen der Theologie und der Naturwissenschaft vorzudringen.

Die religiöse Anknüpfung: Neben der soeben aufgezeigten Abhängigkeit der Theologie von der Naturwissenschft, die teilweise aus einem Gefühl der Dominanz und Mächtigkeit der Naturwissenschaft resultiert, gibt es auch eine Strömung, die sich der Ebenbürtigkeit mit der Naturwissenschaft bewußt ist, und die von einem starken ontologischen Denken aus in einen echten Dialog mit den Naturwissenschaften zu kommen versucht. Hier ist vor allem Paul Tillich mit seiner Methode der Korrela$\operatorname{tion}^{24} \mathrm{zu}$ nennen. Nach Tillich ist es die Aufgabe der Theologie, die in der menschlichen Existenz liegenden Fragen zu erheben und ebenso die in der göttlichen Selbstbekundung liegenden Antworten in Richtung auf die Fragen zu formulieren, die in der menschlichen Existenz liegen ${ }^{25}$. Dabei ist der Mensch die Frage nach sich selbst, ehe er eine Frage gestellt hat ${ }^{26}$. Zur Analyse des Menschen und damit seiner Fraglichkeit dient das Material, das die menschliche Selbstinterpretation auf allen Kulturgebieten verfügbar gemacht hat. Dieser Wissensstoff wird dann vom Theologen in bezug auf die von der christlichen Botschaft gegebene Antwort geordnet ${ }^{27}$. Da der Kern der Antwort der logos des Seins ist, so wie er sich

Eine Antwort an Helmut Gollwitzer, in: Zeit und Geschichte, Dankesgabe an Rudolf Bultmann zum 80. Geburtstag, Tübingen 1964, 412 und 416.

21 Vgl. Braun, Gottes Existenz, 404.

22 Diese fragliche Interpretation Brauns bietet H.-G. Geyer, Gottes Sein als Thema der Theologie, in: Verkündigung und Forschung, Bh zur EvTh, 11. Jg., München 1966, 6.

23 Vgl. etwa John A. T. Robinson, Eine neue Reformation?, München 1965, 119; H. Cox, Stadt ohne Gott, Stuttgart-Berlin 1966, 280 f., und W. Hamilton, The New Essence of Christianity, New York 1961, 65.

24 Vgl. P. Tillich, Systematische Theologie, Bd. I, Stuttgart 1955, 73-80.

25 So Tillich, Systematische Theologie, Bd. I, 75.

28 So Tillich, Systematische Theologie, Bd. I, 76.

27 Ahnlich Tillich, Systematische Theologie, Bd. I, 77. 
in Christus kundgetan hat, kann er nichit durch die Existenzanalyse selbst abgeleitet werden ${ }^{28}$. Gerade hier aber lieegt die Schwäche des sonst so überzeugenden Systems Tillichs, denn nach Tillich ist ja der Gegenstand des Philosophen der universale logos und wo immer der universale logos am Werk ist, stimmt er mit der christlichen Botschaft überein ${ }^{20}$. Antwort und Frage müssen sich also sowieso im Innerrsten entsprechen, denn sie bergen in sich den gleichen logos. So sind Antwcort und Frage im Grunde genommen eine Tautologie. Tillich will zwar eiin echtes Gespräch zwischen Theologie und allen Wissensgebieten einschliceßlich der Naturwissenschaft entfalten und dabei die Eigenständigkeit dler Theologie gegenüber den Wissenschaften und umgekehrt nicht aufgebren, aber trotz der Tautologie von Frage und Antwort, zu der Tillich effelktiv kommt, fragt die Welt nicht so, wie es Tillich will ${ }^{30}$, denn sie kann (es gar nicht. Der sich in Christus offenbarende logos ist nämlich nach derm christlichen Glauben deswegen von jedem in der Welt sichtbaren loggos unterschieden, weil Christus weder geschaffen noch sündhaft ist. $\mathrm{Da}$ T.illich diese ontologische Differenz $\mathrm{zu}$ wenig betonte, konnte er die Welt »»religiös" interpretieren und mit ihr ins Gespräch kommen.

Das dimensionale Verbältnis: Der :mangelnden Unterscheidung von Gott und Welt versuchte Karl Heim durrch den Begriff des dimensionalen Verhältnisses zu begegnen ${ }^{31}$. Die durch cdas heutige Naturbild verursachten Erschütterungen des Glaubens habeen nach Heim die religiöse Frage nach Gott verstummen lassen. Doch istt damit nicht das Rätsel um die eigene Existenz zur Ruhe gekommen. Auf die Fragen: Woher komme ich? und: Welchen Weg habe ich einzuschlagen?, gibt es keine verbindlichen Antworten, da alle immanenten Struktturen in einer Polarität, in einem Entweder-Oder enden. Auch eine Fluchit zur Naturwissenschaft kann in diesem allgemeinen Relativismus und SSkeptizismus nicht helfen, denn die naturwissenschaftlichen Absoluta vom Materie, Raum, Zeit und Kausalität haben sich als unhaltbar erwiesen und sind relativ geworden. Will man die Unsicherheitskomponente im menschlichen Leben ausschalten, so

23 So Tillich, Systematische Theologie, Bd. I, 78;.

2) Tillich, Systematische Theologie, Bd. I, 37: Es gibt nichts im Himmel und auf Erden noch jenseits davon, dem sich der Phiilosoph unterwerfen müßte außer dem universalen logos des Seins, wie er sich ihm irn der Erfahrung mitteilt «... daß, wo immer der logos am Werk ist, er mit der chrristlichen Botschaft übereinstimmt $\alpha$.

31 Daß Tillich die Welt gegen ihren Willen relligiös deutete, stellte schon Bonhoeffer fest (vgl. Bonhoeffer, Widerstand, 219). Dem Unterschied zwischen dem ursprünglichen Sein Gottes und dem geschaffenen Seinı der Welt macht besonders A. E. Loen in seinem vorzüglichen Buch "Säkularisationı, Von der wahren Voraussetzung der angeblichen Gottlosigkeit der Wissenschaft «i, München 1965, 223, gegen Tillich geltend.

3. Vgl. zu diesem Abschnitt besonders K. Heim:, Die Wandlung im naturwissenschaftlichen Weltbild, Hamburg 19543; K. Heim, Der christliche Gottesglaube und die Naturwissenschaft, Hamburg 1953², und H. Sichwarz, Das Verständnis des Wunders bei Heim und Bultmann, Stuttgart 1965, bes. S. 16-33 und 55-69. 
gibt es nur die Zuflucht zum überpolaren Raum, zum Ursein Gottes, das sich uns im Glauben auftut. Von dort her läßt sich der feste Halt für die Bewältigung unseres vorfindlichen, polaren Raumes und unserer polaren Existenz gewinnen. Heim hat sich dabei in fairer und erstaunlich gründlicher Weise mit den neuesten Erkenntnissen der Naturwissenschaft auseinandergesetzt und ist $\mathrm{zu}$ dem berechtigten Schluß gekommen, daß sie uns keine letzte Weisung geben können. Damit hat er mit jeglichem Wissenschaftsaberglauben aufgeräumt. Aber er hat gleichzeitig mit der Frage nach der letzten Weisungsvollmacht die Naturwissenschaft auf etwas befragt, worauf sie nicht antworten kann. Zudem ist das dimensionale Verhältnis zwischen Theologie und Naturwissenschaft, das Heim zu Recht betonte, nicht das einzig mögliche dimensionale Verhältnis. Jeder "transzendenten « Weltanschauung müßte, wenigstens phänomenologisch gesehen, gleiche Gründung in einem ähnlichen »überpolaren Urraum« zuzubilligen sein, wie dem christlichen Glauben. Damit wäre aber der "überpolare Urraum «, zu dem man nur im Glauben Zugang hat, letztlich wieder polar.

\section{Prinzipien der Theologie und der Naturwissenschaft}

Damit wir nicht auch an die Naturwissenschaft wie Heim Fragen stellen, die sie nicht beantworten kann, oder die, wie die Fragen Tillichs, nur Scheinfragen sind, müssen wir uns jetzt den Prinzipien von Theologie und Naturwissenschaft zuwenden, denn jede Verhältnisbestimmung muß unweigerlich fehlgehen, wenn man nicht zuerst nach Gründung und Intention der zueinander in Beziehung zu setzenden Größen fragt. Bei der Naturwissenschaft sind dabei drei Bestrebungen kennzeichnend, nämlich Interrelation, Objektivation und Neutralität. Bei der Theologie hingegen scheinen uns Glaubensgestalt und Wirklichkeitsbestreben kennzeichnend.

Interrelationsbestreben der Naturwissenschaft: Das Interrelationsbestreben der Naturwissenschaft zeigt sich darin, daß jedes beobachtetes Phänomen durch die Koordination von Raum und Zeit und durch die Kausalabfolge in ein eindeutiges Verhältnis zu anderen beobachtbaren Phänomenen gebracht wird. Dabei ist allerdings vorausgesetzt, daß man kein Phänomen durch diese Koordinaten selbst eindeutig bestimmen kann, sondern nur durch das in ihnen ausgedrückte Verhältnis zu anderen Phänomenen. Das heißt, daß die Koordinaten keine absoluten Größen an sich sind, sondern nur relative Größen im jeweiligen Bezug zwischen den Phänomenen.

So waren zwar für Kant Raum und Zeit noch völlig verschiedene Begriffe, doch erkannte er sie schon als Formen der sinnlichen Anschauung $^{32}$. Einstein hat in der Relativitätstheorie diese Idealisierung von

32 Vgl. E. Schrödinger, Geist und Materie, Braunschweig 1959, 59, und I. Kant, Kritik 
Raum und Zeit weitergeführt ${ }^{33}$, indem er Raum, Zeit und Materie zu einer unlösbaren Einheit verknüpfte. Da alle drei Größen von dem jeweiligen Bezugssystem abhängig sind, erweist sich der Begriff eines Alters des Universums im Sinne einer absoluten Weltzeit für sinnlos ${ }^{34}$. Davon wird natürlich nicht die Altersbestimmung von Objekten innerhalb des Universums betroffen, also etwa eines Gebirges oder des Mondes. Mit dem Begriff der Vergangenheit kann man so die Gesamtheit all der Momente innerhalb unseres Universums bezeichnen, die prinzipiell erfahrbar sind, während der Begriff der Zukunft die Gesamtheit all der Momente beinhaltet, die wenigstens im Prinzip noch beeinflußbar sind ${ }^{35}$. Die Gegenwart aber entzieht sich der wissenschaftlichen Beschreibung, denn was man unter dem Begriff der Gegenwart bezeichnet, ist im Augenblidk des Beobachtens schon Vergangenheit. Das Beobachten beschränkt sich damit notwendigerweise auf eine Sequenz von Vergangenem ${ }^{36}$. So stellt die Kausaldeutung keinen Bezug zwischen Gegenwärtigem und Vergangenem oder Zukünftigem her, sondern nur zwischen Vergangenem und anderem Vergangenem oder in pragmatischer Spekulation zwischen Vergangenem und Zukünftigem.

Das bedeutet auch ein neues Verständnis der Naturgesetze. So sind Naturgesetze nicht Ergebnis sondern Grundvoraussetzung jeder naturwissenschaftlichen Forschung ${ }^{37}$. Dies zeigt sich etwa am Prinzip des Aktualismus $^{38}$, das besagt, daß alle hier und jetzt gültigen Gesetzmäßigkeiten (zB $\mathrm{c}$ als größtmögliche Geschwindigkeit) überall und $\mathrm{zu}$ allen Zeiten gelten sollen. Hätten $\mathrm{zB}$ im Weltall in großen Entfernungen und vor hinreichend langen Zeiten andere Gesetzmäßigkeiten gegolten als die von uns festgestellten, dann könnten wird nichts über den Zustand und die Entstehung des Kosmos aussagen und erschließen. Aber auch auf unserer Erde ist die Geltung der Naturgesetze nicht zu verifizieren, denn der Ubergang von der experimentell nachprüfbaren Behauptung: "Es gibt Dinge oder Vorgänge von der Art a" zu dem Naturgesetz: "Alle Dinge oder Vorgänge von der Art a haben die Eigenschaften $b$ « wird durch noch so viele nachgeprütte Dinge oder Vorgänge von der Art a nur wahrschein-

der reinen Vernunft, in: I. Kant, Werke, Bd. II, hg. v. W. Weischedel, 1956 (Insel Verlag), 96.

33 So auch Schrödinger, Geist 62.

34 Vgl. dazu G. Frey, Erkenntnis und Wirklichkeit, Philosophische Folgerungen der modernen Naturwissenschaften, Stuttgart-Berlin-Köln-Mainz 1965, 63.

35 So W. Heisenberg, Physics and Philosophy, The Revolution in Modern Physics, London 1958, $102 \mathrm{f}$. Diese Feststellung hat natürlich eminent theologische Konsequenzen.

36 Vgl. Heisenberg, Physics, 54, wo er das an Hand der Atomphysik ausführlich erläutert.

37 So auch G. Stammler, Was ist eigentlich ein Naturgesetz?, KuD, 3. Jg., Göttingen 1957, 11.

${ }^{38}$ Vg! dazu Frey, Erkenntnis, 59. 
licher aber nicht wahr ${ }^{30}$. Ein weiterer Hinderungsgrund für die Verifizierung von Naturgesetzen ist die Meßungenauigkeit. "Nehmen wir zB an, daß die Beschleunigung eines freifallenden Körpers nicht konstant, daß sein Weg nicht eine zum Erdmittelpunkt gerichtete Gerade sei; daß die Beschleunigung sehr schnell um einen Mittelwert schwanke, die Falllinie eine Zickzacklinie darstelle, und daß alle diese Anderungen des bekannten Gesetzes unterhalb der Meßgenauigkeit liegen. Kein Experiment könnte sie daher widerlegen. Aber eben dies zeigt, daß auch keine Erfahrung beweist, daß das Fallgesetz wabr ist " ${ }^{40}$. Ahnliches zeigt sich auch am Gesetz der Halbwertzeit. Bei einer genügend großen Menge ist seine Geltung unbestritten, während es bei einem einzelnen radioaktiven Atom sinnlos wird. Naturgesetze sind demnach apriorische Prinzipien, die in bestimmten Grenzen eine Ordnung beschreiben, nach denen uns Naturphänomene erschienen sind und wahrscheinlich fernerhin erscheinen.

Objektivierungsbestreben der Naturwissenschaft: Haben sich Raum, Zeit, Kausalität und Naturgesetzlichkeit als relationale, objektbezogene Größen erwiesen, so erhebt sich die Frage, inwieweit dem Objekt dann noch ein an sich seiender Objektscharakter eignet. Auch hier hat die moderne Atomphysik zu einer Neuorientierung geführt. Zunächst wurde durch Einstein theoretisch und durch die Atomphysik experimentell festgestellt, daß Materie und Energie ineinander konvertierbar sind ${ }^{41}$. Die Materie kann demnach nicht mehr als Absolutum betrachtet werden. Weit wichtiger für den Begriff der Objektivität ist aber, daß Objekte jeweils nur mögliche Objekte eines Subjekts sind ${ }^{42}$. So entscheidet nach der Heisenbergschen Unschärferelation der Beobachter darüber, inwieweit der "Fehlerquotient " $^{43}$ der Orts- und Impulsbestimmung eines Elektrons, also eines Grundbausteines des Atoms, der den Betrag des Planckschen Wirkungsquantums h nicht unterschreiten darf, zugunsten einer genaueren Orts- oder Impulsbestimmung verschoben werden soll. Damit wird die Art des Ergebnisses einer Messung durch die Versuchsanordnung oder den Willen des Beobachters präjudiziert. Wenn wir uns den Elementarteilchen selbst zuwenden, wird dieser Einfluß des Beobachters noch deutlicher. So haben die Elementarteilchen und das Licht Doppelnatur, dh unter bestimmten Bedingungen zeigen das Licht und die Elementarteilchen ausgesprochenen Wellencharakter, während sie unter anderen Bedin-

30 Ahnlich auch A. March, Die physikalische Erkenntnis und ihre Grenzen, Braunschweig 19602, 31.

40 Dieses treffende Beispiel bringt $\mathrm{K}$. Hübner, Beiträge zur Philosophie der Physik (Rezensionen), Phil. Rundschau, Bh 4, 11. Jg., Tübingen 1963, 98.

41 Vgl. dazu ausführlicher Heisenberg, Physics, 139.

42 Vgl. Hübner, Beiträge, $75 \mathrm{f}$,, der an dieser Stelle die entsprechende Meinung Weizsädkers zitiert.

13 Wir sind natürlich mit March, Physikalische Erkenntnis, 70, der Meinung, daß das Produkt von $\triangle p \cdot \triangle q$ kein Produkt von Fehlern im herkömmlichen Sinne ist, sondern nur von undefinierten Größen. 
gungen Korpuskelcharakter haben"4. Das Eigentümliche dabei ist aber, $\mathrm{daß}$ es sich um komplementäre Charaktere handelt, dh das Auftreten des einen Charakterzugs schließt das gleichzeitige Auftreten des anderen am gleichen, beobachteten Phänomen aus. Das heißt nun, daß die unmittelbar sinnliche Wahrnehmung eines Phänomens nichts über sein objektives Wesen aussagt $t^{45}$, oder anders ausgedrückt: die naturwissenschaftliche Forschung hat es stets ausschließlich mit den Phänomenen zu tun, aber nicht mit einem etwaigen Ding an sich ${ }^{46}$. Da nun im Gegensatz zu der bei Descartes noch möglichen Argumentationsweise - naturwissenschaftlich gesprochen - die Wirklichkeit Gottes nicht mehr die Wirklichkeit der Außenwelt garantieren kann ${ }^{47}$, ist die Existenz der Außenwelt als Ding an sich unbeweisbar ${ }^{48}$. Die Erfahrung der phänomenalen Welt ist also immer zum Teil von uns selbst erzeugt, so daß es nicht mehr möglich ist, von einem Objekt an sich zu reden, sondern nur vom Objekt eines Subjekts oder von einer subjektiven Objektivität". Der Mensch macht zwar philosophisch gesprochen einerseits das Seiende zum Gegenstand ${ }^{\text {so }}$, aber er wird als der Verstehende selbst in das zu erkennende Geschehen mit hineinbezogen ${ }^{51}$.

Neutralitätscharakter der Naturwissenschaft: Es wäre nun kurzschlüssig, wollten wir aus der Interrelation von Raum, Zeit, Kausalität und Materie, aus der Unbeweisbarkeit der Naturgesetze und aus der subjektiven Objektivität den Schluß ziehen, daß die naturwissenschaftliche Forschung in einem haltlosen Relativismus endigt. Vielmehr ist der Naturwissenschaft ein ihr eigentümliches Neutralitätsstreben eigen, das vom Bemühen getragen ist, innerhalb gewisser Grenzen verbindliche Aussagen zu machen. So sind etwa trotz der subjektiven Objektivität die in Erscheinungsgesetze geordneten beobachtbaren Phänomene jederzeit nachprüfbar und haben bis jetzt immer zu denselben Resultaten geführt. Das

44. Vgl. dazu March, Physikalische Erkenntnis, 99.

45 So auch Schrödinger, Geist 76.

46 Ahnlich March, Physikalische Erkenntnis, 20.

$47 \mathrm{Vgl}$. R. Descartes, Meditationes de prima philosophia, hg. v. L. Gäbe, Hamburg 1959, 128.

48 Frey, Erkenntnis, 169, betont mit Recht, daß die Schlußfolgerung aber sinnlos wäre, $\mathrm{da} ß$ es kein wirklich Seiendes gibt. Nur kann für die Existenz dieses Seienden kein eigentlicher Beweis erbracht werden.

49 Diese Interrelation von Subjekt und Objekt ist wohl naturwissenschaftlich allgemein akzeptiert. Vgl. dazu etwa: Frey, Erkennntnis, 96 f.; Hübner, Beiträge, 75; und M. Plandk, Where is Science Going?, New York 1932, 95.

50 Ahnlich auch M. Heidegger, Holzwege, Frankfurt am Main 19634, 236 und 242.

51 Ahnliches meint auch H.-G. Gadamer, Wahrheit und Methode, Grundzüge einer philosophischen Hermeneutik, Tübingen 1960, 465, wenn er sagt: «Wir sind als Verstehende in ein Wahrheitsgeschehen einbezogen und kommen gleichsam zu spät, wenn wir wissen wollen, was wir glauben sollen $\kappa$. Nur muß man in unserem Falle bezüglich des Wahrheitsbegriffes sehr vorsichtig sein. Wahr ist dabei nur, daß uns etwas so erscheint, wie es uns erscheint, wobei dieses "wie es uns erscheint * auch als Erscheinung für andere nachvollziehbar ist. 
Neutralitätsbestreben besagt aber auch, daß die Naturwissenschaft sich bewußt ist, nur innerhalb gewisser Grenzen verbindliche Aussagen machen zu können, und daß diese Aussagen in ihrer Verbindlichkeit selbst begrenzt sind. Eine dieser Grenzen ist die Beschränkung auf Aussagen über beobachtbare Phänomene. Die Naturwissenschaft erklärt die Phänomene der Welt, indem sie sie auf andere, beobachtbare Phänomene zurückführt und sie mit denselben kausal oder analogisch verknüp $\mathrm{ft}^{32}$. Das heißt philosophisch ausgedrückt, daß das Sein des Seienden nicht Gegenstand des naturwissenschaftlichen Erkennens ist, oder theologisch gesagt, daß über das Sein oder das Wirken Gottes in der Welt a priori nichts ausgesagt wird, da Aussagen über das Sein oder das Wirken Gottes Qualitätsaussagen sind und deshalb nicht Gegenstand des naturwissenschaftlichen Erkennens sein können. Die Naturwissenschaft kann nicht etwa eine Gottlosigkeit als Ergebnis zeitigen, die sie als Methode bereits voraussetzt ${ }^{53}$. Das heißt natürlich nicht, daß die methodische Gottlosigkeit nicht sehr oft zu einer grundsätzlichen Gottlosigkeit führen kann ${ }^{54}$, doch kann man die Naturwissenschaft dafür nicht verantwortlich machen ${ }^{55}$. Man muß sogar noch weiter feststellen, daß die Naturwissenschaft auf Grund ihrer Prinzipien zu keinen theologischen oder religiösen Ergebnissen kommen kann, obwohl alle naturwissenschaftlichen Ergebnisse religiös oder theologisch deutbar sind ${ }^{36}$. Doch damit sind wir schon bei den Prinzipien der Theologie, denen wir uns jetzt zuwenden müssen.

Wenn wir nach den Prinzipien der Theologie fragen, so heißt das nicht, daß wir nach dogmatischen Grundprinzipien fragen sondern wir wollen uns zunächst rein phänomenologisch mit der Grundgestalt christlicher Theologie befassen. Dabei sind für die christliche Theologie ihre Glaubensgestalt und ihr ganzheitliches Wirklichkeitsverständnis konstitutiv. Die christliche Theologie verzichtet von vornherein auf die Behauptung, daß ihre Prinzipien rational evident und durch Experimente nachprüfbar sind. Sie beruft sich vielmehr auf eine Glaubensentscheidung. Zum

s2 So sagt etwa W. Heisenberg, Philosophic Problems of Nuclear Science, London 1958, 18: Science has two tasks: to pass on an understanding of nature, thus enabling man to make nature serve his own purpose, and to indicate to man his appropriate position in nature through a real insight into its inter-relations $*$.

$53 \mathrm{Vgl}$. dazu auch ausführlicher R. Bultmann, Der Gottesgedanke und der moderne Mensch, GuV Bd. IV, Tübingen 1965, 115, und B. Bavink, Science and God, New York 1934, 162.

54 Diese Konsequenz betont besonders H. Benkert, "Ohne Christ wäre ich Atheist火, Zur Frage der natürlichen Gotteserkenntnis, EvTh, 18. Jg., München 1958, 458.

$35 \mathrm{Vgl}$. Frey, Erkenntnis, 151. Sehr instruktiv ist in dieser Hinsicht die Untersuchung von E. LeRoy, Jr., Religious Beliefs of American Scientists, Philadelphia 1952, in der er zu dem Ergebnis kommt, daß bei den amerikanischen Naturwissenschaftlern dieselbe Streuungsbreite von religiösen Haltungen vorliegt wie bei anderen Berufsgruppen (aaO, $146 \mathrm{f}$.).

56 E. L. Mascall, Christian Theology and Natural Science, New York 1956, 161 f., zeigt das etwa an Hand naturwissenschaftlicher, kosmologischer Theorien. 
andern ist sie bestrebt, die Wirklicchkeit in ihrer Totalität zu verstehen. Sie duldet zumeist nicht, daß ihr Włirklichkeitsverständnis gleichberechtigt neben anderen steht, sondern von ilhrer Glaubensgestalt aus behauptet sie, daß ihr Wirklichkeitsverständnis dias alleingültige ist.

Glaubensgestalt der Theologiie: Die Glaubensgestalt der Theologie besagt nicht, daß sich die Theologie: auf Spekulation gründet, sondern daß ihr Grund bereits einer glaubensmäßigen Interpretation von historisch Vorgegebenem entspringt. Das hisstorisch Vorgegebene ist dabei Person und Schicksal Jesu von Nazareth, und die glaubensmäßige Interpretation dessen zeigt, daß sich in Person undl Schicksal Jesu Gott offenbart und uns seine Gnade zugesagt hat, indem Jeesus selbst die Offenbarung Gottes war und sie damit im gegenwärtigen Christus für uns ist. Diese Interpretation ist keine Spekulation, sondern Naachvollzug des glaubensmäßigen $\mathrm{Be}-$ kenntnisses, das sich im neutestamentlichen Kerygma niedergeschlagen hat. Damit ist die Glaubensgestaltt der Theologie zwar im historischen Ereignis der Geschichte Jesu gegrünıdet; doch sind nicht etwa isolierte und objektivierbare Fakten der Geschichte Jesu der Glaubensgrund ${ }^{37}$, sondern zwischen Jesus und dem Kerygma coder der Verkündigung des Glaubens an Jesus steht das Faktum der Aufrerstehung ${ }^{58}$. Die Auferstehung ist das Ende des historischen Jesus und der Beginn des verkündigten Christus; durch sie wird der Verkündiger zuım gegenwärtig Verkündigten. Damit wird der historische Jesus nicht etrwa gleichgültig, denn er muß als der Bezugspunkt des Glaubens stets feestgehalten werden ${ }^{50}$. Uberdies erhob bereits Jesus einen Anspruch, der iüber das phänomenologisch Feststellbare hinausgeht und nur glaubenssmäßig zu erfassen ist. So kam etwa Rudolf Bultmann zu dem Ergebmis, daß Jesu Entscheidungsruf eine Christologie impliziert, während Ernst Fuchs das dahin abwandelte, daß Jesu Verhalten eine Christologiie impliziert ${ }^{80}$. Hans Conzelmann hat das in anderer Form konkretisiert, wwenn er sagt: "Sowohl in der Kosmologie (der Lehre vom Walten Gottees) als in der Eschatologie (der Lehre vom Kommen Gottes) als in der Eithik (der Lehre vom Willen Gottes) stoßen wir auf den Tatbestand einer indirckten Christologie und diese hat jeweils den Sinn, den Hörer unmitttelbar mit diesem Gott zu konfron-

Di Dagegen wendet sich berechtigterweise (G. Ebeling, Die Frage nach dem historischen Jesus und das Problem der Christologie, ZThK, 56. Jg., Bh 1, Tübingen 1959, 30. Wenn er aber den Grund des Glauboens allein in Jesus als den Zeugen des Glaubens findet, so klingt das sehr nach eineer mittelalterlichen Imitatio-Theologie. Hier scheint doch Jesus zu sehr als Vorbild betont zu sein, aber nicht als analogieloser Sohn Gottes, der uns eben trotz allem ksein Zeuge des Glaubens sein kann, weil wir nicht wie Jesus sondern nur wie Sünder glauben können.

5* So auch H. Conzelmann, Zur Methodee der Leben-Jesu-Forschung, ZThK, 56. Jg., Bh 1, Tübingen 1959, 6 f., dessen Aufsoatz uns zu unsrem Problem sehr instruktiv erscheint.

5) Vgl. Conzelmann, Methode, $8 \mathrm{f}$.

or Vgl. dazu Conzelmann, Methode, 6. 
tieren. Man kann zugespitzt formulieren: Jesus versteht sich als der V'ollzug dieser direkten Konfrontation ${ }^{61}$. So fordert schon der historische Jesus zu einer Glaubensentscheidung auf. Wichtig ist, daß diese indirekte Christologie in eine direkte Christologie umgewandelt wurde, als auf Grund der Ostererscheinungen Jesus als Christ in einer neuen Weise seines Dabeiseins verstanden wurde. Die direkte Christologie hat zwar die indirekte Christologie als eine ihre Voraussetzungen ${ }^{62}$, gründet aber andererseits in einem neuartigen Verständnis des Jesusgeschehens, das durch die Auferstehung ermöglicht wurde. Christus wurde nämlich als der erkannt, der er war und ist. Durch die Auferstehung verschiebt sich die Glaubensentscheidung von dem Ja zum Anspruch des historischen Jesus zum Ja zum Anspruch des gegenwärtigen Christus. Dieses Ja impliziert kein Ja zu einem in dieser Welt beobachtbaren Phänomen und damit zu einem von der Naturwissenschaft zu untersuchenden Gegenstand. Wohl aber impliziert es einen Rüdkbezug auf die historische Person Jesu ${ }^{\text {bs }}$ als einem objektivierbaren Phänomen. In diesem Rückbezug auf die Welt der Phänomene liegt das andere Grundcharakteristikum der christlichen Theologie, nämlich ihr Wirklichkeitsbestreben.

Das ganzbeitliche Wirklichkeitsverständnis der Theologie: In dem Bekenntnis zu Jesus Christus als dem, der vere deus et vere bomo war, liegt die Möglichkeit der Theologie begründet, Gott, Welt und Mensch zueinander in eine Beziehung zu setzen ${ }^{64}$, die die ganze Wirklichkeit des Glaubens und der Welt umfaßt. Das Wirklichkeitsbestreben der Theologie ist also darin zu sehen, an Hand des einmaligen Handelns Gottes in und an Jesus sein Handeln in und an dieser Welt auszusagen und so von Jesus Christus her die Welt als Wirklichkeit Gottes zu verstehen. Ebenso wie jedes Glaubensbekenntnis einen Rüdkbezug auf die historische Person Jesu enthält, kann man als Theologe nicht von Gott oder der Welt an und für sich reden. Redet die Theologie von dem Wirken Gottes, so kann sie sein Wirken an oder in der Welt nicht davon ausnehmen ${ }^{65}$. Alle Aussagen darüber sind aber nicht nur Aussagen über Gott, sondern auch über die Welt. Man kann sogar sagen, daß das Reden von Gott und von seiner Offenbarung auf nichts anderes abzielt, als in rechter Weise von der W'elt, von der Geschichte und vom Menschen, also von der Totalität unserer

\footnotetext{
61 Siehe Conzelmann, Methode, 12.

$62 \mathrm{Vgl}$. Conzelmann, Methode, 13.

63 Vgl. dazu H. Conzelmann, Jesus von Nazareth und der Glaube an den Auferitandenen, in: Der historische Jesus und der kerygmatische Christus, hg. v. H. Ristow und K. Matthiae, Berlin 19612, 195, der zu dem Ergebnis kommt, daß in sllen Glaubensformulierungen ein Rückbezug auf die geschichtliche Person Jesu enthalten * ist.

o4 Dies betont auch G. Ebeling, Theologie und Wirklichkeit, ZThK, 53. Jg., Tübingen 1956, 382.

os Zum Folgenden vgl. W. Elert, der christliche Glaube, Hamburg 19564, 247.
} 
Wirklichkeit, zu reden ${ }^{66}$. Darunter ist natürlich nicht zu verstehen, daß die Theologie Aussagen über die Wirklichkeit in Analogie zur Naturwissenschaft macht, sondern sie erhebt die von der Naturwissenschaft erforschten Phänomene zur Wirklichkeit, indem sie sie auf Gott bezieht. So entlarvt sie die in der Naturwissenschaft herrschende Gottlosigkeit als methodische Gottlosigkeit und trägt damit dazu bei, daß über den Phänomenen nicht Gott als der Grund der Phänomene vergessen wird. Indem die ganze phänomenale Welt auf das einmalige Handeln Gottes in und an Jesus bezogen wird, beantwortet die Theologie auch die Sinnfrage dieser Welt. Sie behauptet und erkennt im Glauben an das Christusgeschehen, daß Gott in den Phänomenen sub contraria specie wirksam ist. Sie erkennt und bekennt aber auch, daß die Welt auf eine Umwandlung und Neuschöpfung zudrängt, die in der Auferstehung Christi angebrochen ist. Die Glaubensgestalt der Theologie steht also nicht im luftleeren Raum, den es bekanntlich nicht gibt, sondern sie umfaßt die ganze Wirklichkeit in allen ihren Bezügen, weil sie das Wirken Gottes auf alle diese Bezüge bezieht. Dabei ist der Gott, der in den Phänomenen der Welt erkannt wird, kein anderer als der, der in Jesus Christus war. Er ist weder hier noch dort deutlicher zu erkennen, doch hat er sich allein in Jesus Christus offenbart und seinen Liebeswillen uns kundgetan und ist darum von dort aus auf alle anderen Phänomene zur Wirklichkeitserkenntnis zu beziehen und nicht umgekehrt. In diesem Wirklichkeitsbestreben, bei dem die ständig wechselnden Phänomene der Welt jeweils auf das Christusgeschehen bezogen werden und von daher zu einer ganzheitlichen Wirklichkeitsschau interpretiert werden, wird schon etwas von der Interdependenz von Theologie und Naturwissenschaft deutlich, der wir uns abschließend zuwenden müssen.

\section{Die Interdependenz von Theologie und Naturwissenschaft}

Wenn wir das gegenseitige Verhältnis von Theologie und Naturw:ssenschaft $\mathrm{zu}$ bestimmen versuchen, so scheint uns im gegenseitigen Verhaltnis sowohl eine koordinative als auch eine dimensionale Wirklichkeitserfassung vorzuliegen.

Die koordinierte Wirklichkeitserfassung: Im Wirklichkeitsbestreben der Theologie zeigte sich schon, daß Theologie und Naturwissenschaft keine getrennten Bereiche sind, die nichts miteinander $\mathrm{zu}$ tun haben, sondern der Rückbezug des Glaubens auf die historische Person Jesu und die diraus resultierende Interpretation der Gesamtwirklichkeit impliziert notwendigerweise, daß Theologie und Naturwissenschaft koordinativ nibeneinander wirken. So ergründet die Naturwissenschaft die beobacht-

6' Ahnlich auch Ebeling, Theologie und Wirklichkeit, 379. 
baren Phänomene der Welt einschließlich auch dessen, was an der historischen Person Jesu wissenschaftlich zu ergründen ist. Die Naturwissenschaft liefert also die Strukturen der beobachtbaren Welt. Die Theologie hingegen deutet diese Strukturen auf Grund des Christusereignisses; sie bestätigt damit gleichsam die Wirklichkeit der Phänomene, indem sie sie auf die Wirksamkeit und Schöpferkraft Gottes bezieht. Sie gibt ihnen einen Sinn, der jenseits der Phänomene selbst liegt, indem sie auf ihren Anfang und ihre Erfüllung hinweist. Dabei erkennt sie in diesen Phänomenen die Unergründlichkeit Gottes, die letztlich von dem Liebeswillen bestimmt ist, den Gott uns in Jesus Christus offenbart hat.

Der christliche Glaube beeinflußt damit nicht die Ergebnisse der naturwissenschaftlichen Forschung, sondern er interpretiert sie ${ }^{67}$. So ist kein sacrificium intellectus zu Gunsten des christlichen Glaubens nötig ${ }^{68}$, sondern dieser Glaube ist - phänomenologisch gesehen - eine Möglichkeit neben anderen; für den aber, der von der Wirklichkeit des christlichen Glaubens herkommt, wird er zur einzigen Möglichkeit einer sinnvollen Welterfassung und Lebensgestaltung. Für den christlichen Glauben zerstört die Wissenschaft nicht das Geheimnis des Wirkens Gottes, sondern in jedem neuen Forschungsergebnis wird dessen unausschöpfbare Tiefe erneut und verstärkt deutlich ${ }^{80}$. Vom Glauben her gesehen spiegelt sich in der Herrlichkeit und der erschreckenden Rätselhaftigkeit der Welt die Herrlichkeit Gottes wieder und verpflichtet so den Wissenschaftler zur Ehrfurcht vor der Welt als der Schöpfung Gottes. Der verantwortliche Gebrauch der Schöpfungsgüter und der Dank und die Freude über die Schöpfungsgüter sind damit die sachgemäße Reaktion auf diese Interpretation der Phänomene der Welt.

Die dimensionale Wirklichkeitserkenntnis: Es wäre nun verkehrt, wollte man daraus folgern, daß der christliche Glaube die naturwissenschaftlichen Ergebnisse ergänzt oder erhöht, oder daß von diesen Ergebnissen her ein Rückschluß auf Gott und auf den christlichen Glauben möglich wäre ${ }^{70}$. Der christliche Glaube gründet in dem, was in den Phänomenen dieser Welt nicht beobachtbar ist, und ist also von anderer Dimension als diese Phänomene und die Naturwissenschaft. Damit ist kein Rückschluß von der Naturwissenschaft her auf Gott möglich. Der christliche Glaube ist von der Naturwissenschaft her weder zu begründen noch zu leugnen ${ }^{71}$. Wird solches versucht, so handelt es sich um eine Grenzüber-

67 Ahnlich auch M. H. Hartshorne, The Promise of Science and the Power of Faith, Philadelphia 1958, 119.

68 Dies betont besonders W. Künneth, Schöpfungsglaube und Naturwissenschaft, in: Paideia, Erziehung und Bildung heute, I, Villingen 1965, 143.

6o Vgl. W. Weidlich, Fragen der Naturwissenschaft an den christlichen Glauben, ZThK, 64. Jg., Tübingen 1967, 245.

70 So auch S. M. Daedke, Gott der Schöpfer, in: Gott heute, hg. v. N. Kutschki, MainzMünchen 1967, $58 \mathrm{f}$.

71 So betont E. Schlink, Thesen über Theologie und Naturwissenschaften, EvTh, 7. Jg., 
schreitung der Naturwissenschaft, die von den Prinzipien der Naturwissenschaft her verboten ist. Genauso illegitim wäre eine Korrektur naturwissenschaftlicher Ergebnisse vom christlichen Glauben her. $\mathrm{Da}$ es sich um ein dimensionales Verhältnis handelt, können Theologie und Naturwissenschaft auch nicht beziehungslos nebeneinander existieren. Weil Gott als Gott und als Schöpfer bekannt wird, ist vielmehr das, was vom christlichen Glauben her aussagbar und von der Naturwissenschaft her erkennbar ist, auf die eine Wirklichkeit zu beziehen, in und mit der wir leben ${ }^{72}$. Es besteht dann auch keine Schwierigkeit mehr darin, daß das Welthandeln Gottes nicht als solches sinnvoll erkennbar gemacht werden $\mathrm{kann}^{73}$, denn das Welthandeln Gottes kann nur aus der Glaubenshaltung heraus bezeugt werden, die vom Liebeshandeln Gottes überzeugt ist, das sich im Christusgeschehen vollzogen und offenbart hat.

München 1947/48, 94, daß die Lehraussagen der Theologie und die exakten Ergebnisse der Naturwissenschaften einander nicht widersprechen können.

72 Ahnlich auch E. LeRoy Long, Jr., Religious Beliefs, 148.

i3 Vgl. dazu J. Dillenberger, God Hidden and Revealed, The Interpretation of Luther's deus absconditus and its Significance for Religious Thought, Philadelphia 1953, 177, und C. H. Ratschow, Das Heilshandeln und das Welthandeln Gottes, Gedanken zur Lehrgestaltung des Providentia-Glaubens in der evangelischen Dogmatik, NZSTh, 1. Jg., Berlin 1959, 80. 